\title{
Implementation of the American College of Surgeons Oncology Group Z1071 Trial Data in Clinical Practice: Is There a Way Forward for Sentinel Lymph Node Dissection in Clinically Node-Positive Breast Cancer Patients Treated with Neoadjuvant Chemotherapy?
}

\author{
Elizabeth A. Mittendorf, MD, $\mathrm{PhD}^{1}$, Abigail S. Caudle, $\mathrm{MD}^{1}$, Wei Yang, $\mathrm{MD}^{2}$, Savitri Krishnamurthy, $\mathrm{MD}^{3}$, \\ Simona Shaitelman, $\mathrm{MD}^{4}$, Mariana Chavez-MacGregor, $\mathrm{MD}^{5}$, Wendy A. Woodward, $\mathrm{MD}, \mathrm{PhD}^{4}$, \\ Isabelle Bedrosian, MD' ${ }^{1}$, Henry M. Kuerer, MD, PhD ${ }^{1}$, and Kelly K. Hunt, MD $^{1}$ \\ ${ }^{1}$ Department of Surgical Oncology, The University of Texas MD Anderson Cancer Center, Houston, TX; ${ }^{2}$ Department of \\ Radiology, The University of Texas MD Anderson Cancer Center, Houston, TX; ${ }^{3}$ Department of Pathology, The \\ University of Texas MD Anderson Cancer Center, Houston, TX; ${ }^{4}$ Department of Radiation Oncology, The University of \\ Texas MD Anderson Cancer Center, Houston, TX; ${ }^{5}$ Department of Breast Medical Oncology, The University of Texas MD \\ Anderson Cancer Center, Houston, TX
}

\begin{abstract}
For clinically node-positive breast cancer patients receiving neoadjuvant chemotherapy, approximately $40 \%$ will be found to be pathologically node negative. The American College of Surgeons Oncology Group Z1071 trial was therefore conducted to evaluate sentinel lymph node dissection (SLND) in these patients. The trial's primary end point was to determine the falsenegative rate (FNR) among patients with clinical N1 disease in whom at least 2 sentinel lymph nodes (SLNs) were identified. The FNR was $12.6 \%$, which exceeded the prespecified end point of $10.0 \%$. After data publication, our multidisciplinary team discussed the trial results and how we may incorporate the findings into clinical practice. Patient selection and surgical technique are critical. As an example, when dual tracer technique was used, the FNR was $10.8 \%$. Data from the trial presented at the San Antonio Breast Cancer Symposium suggested that the FNR could be improved if a clip was placed in the biopsy-proven positive lymph node and removal of that node during
\end{abstract}

\section{(C) Society of Surgical Oncology 2014}

First Received: 20 March 2014;

Published Online: 20 May 2014

E. A. Mittendorf, $\mathrm{MD}, \mathrm{PhD}$

e-mail: eamitten@mdanderson.org

K. K. Hunt, MD

e-mail: khunt@mdanderson.org
SLND was confirmed. Taking this into consideration, we have proposed an approach to surgical management of the axilla in clinically node-positive patients receiving neoadjuvant chemotherapy termed targeted axillary dissection (TAD). TAD involves placing a clip at the time a lymph node is determined to be positive. After completion of neoadjuvant chemotherapy, the clipped node is localized by using a wire or radioactive seed, and during the SLND procedure, all SLNs and the clipped node are removed. We are currently evaluating the efficacy of TAD in axillary staging after neoadjuvant chemotherapy.

Sentinel lymph node dissection (SLND) is the standard for axillary staging for clinically node-negative breast cancer patients. When the sentinel lymph node (SLN) is negative, axillary lymph node dissection (ALND) can be omitted without a negative effect on overall survival, disease-free survival, or local-regional control, sparing patients the morbidity associated with ALND. ${ }^{1,2}$

The practice of performing SLND in clinically nodenegative patients is supported by single-institution, multicenter, and prospective randomized trials. The National Surgical Adjuvant Breast and Bowel Project (NSABP) B-32 trial randomized 5,611 women to SLND plus ALND or SLND and ALND only if the SLN was positive. Tenyear follow-up data showed no difference in disease-free survival or overall survival between patients with 
SLN-negative disease who underwent ALND versus SLND alone. $^{3}$ Local-regional recurrences were uncommon, occurring in approximately $4 \%$ of patients in both arms of the trial. Axillary recurrences as a first event were rare, occurring in three $(0.2 \%)$ patients in the ALND arm and nine $(0.4 \%)$ in the SLND arm. SLND in clinically nodenegative patients receiving neoadjuvant chemotherapy is supported by numerous retrospective studies showing a high SLN identification rate and false-negative rates (FNRs) comparable to those seen in patients undergoing SLND before chemotherapy. ${ }^{4,5}$ In a study from the MD Anderson Cancer Center that included 575 clinically nodenegative patients undergoing SLND after neoadjuvant chemotherapy, investigators reported an SLN identification rate of $97.4 \%$ and an FNR of $5.9 \%{ }^{6}$ For patients with T2 and T3 tumors, there was a lower rate of positive SLNs in patients receiving neoadjuvant chemotherapy compared with those undergoing surgery first, resulting in fewer patients with clinical T2/T3 tumors requiring ALND after chemotherapy.

Given the benefits of SLND in patients presenting with clinically node-negative disease, clinicians have questioned whether the technique could be applied to patients presenting with node-positive disease who receive neoadjuvant chemotherapy. Up to $40 \%$ of patients treated with neoadjuvant chemotherapy will have a complete response in the axillary nodes. This response rate is even higher in patients with estrogen receptor-negative disease or high-grade tumors and as high as $74 \%$ in those with HER2-positive tumors treated with trastuzumab-based neoadjuvant chemotherapy. $^{7-9}$ Removing lymph nodes with no residual tumor is unlikely to benefit these patients. Initial reports of small series where SLND was performed in patients with clinically node-positive disease who receive neoadjuvant chemotherapy reported FNRs of up to $25 \% .^{10-13}$ Although it was not a primary or secondary end point of the trial, some surgeons performed SLND in addition to completion ALND in patients treated with neoadjuvant chemotherapy in the NSABP B-27 trial. Of 428 patients who had SLND performed, the FNR was $10.7 \% .^{14}$ The cohort included both clinically node-positive and node-negative patients, and there was no significant difference between these groups with respect to the FNR. There was no requirement for biopsy to confirm node-positive disease prior to initiation of chemotherapy. To prospectively study the question of the accuracy of SLND in clinically node-positive patients receiving neoadjuvant chemotherapy, the American College of Surgeons Oncology Group (ACOSOG) completed the Z1071 trial, a phase II study designed to determine the FNR for SLND performed after neoadjuvant chemotherapy in breast cancer patients presenting with pathologically confirmed nodepositive disease.

\section{ACOSOG Z1071}

The ACOSOG Z1071 trial enrolled patients with clinical stage T0-4, N1-2, M0 breast cancer. Axillary disease was confirmed by fine-needle aspiration or core needle biopsy. Patients received neoadjuvant chemotherapy, after which they underwent SLND with ALND. The protocol specified that at least two SLNs be resected, and surgeons were encouraged to use a dual tracer technique with radioisotope and blue dye. SLNs were examined with hematoxylin and eosin, and a positive SLN was defined as metastasis larger than $0.2 \mathrm{~mm}$. The primary aim was to determine the FNR of SLND after chemotherapy in the patients with clinical N1 disease when at least two SLNs were removed. The prespecified criteria for success was a FNR of $10 \%$ or less, consistent with accepted FNRs in patients presenting with clinically negative nodes. ${ }^{7}$

Between July 2009 and June 2011, 756 patients were enrolled. Of these, 663 patients had clinical N1 disease, and 649 completed chemotherapy followed by SLND and ALND. The SLN identification rate was $92.9 \%$. One SLN was identified in 78 patients, leaving 525 patients with two or more SLNs identified for the primary end point analysis. Of these 525 patients, 215 (41\%) had a pathologic complete nodal response. In 39 patients, metastases were not identified in the SLN but were found in the ALND specimen; therefore, the FNR was $12.6 \%$ (90\% Bayesian credible interval, 9.85-16.05\%). ${ }^{7}$ ACOSOG Z1071 investigators concluded that changes in approach and patient selection resulting in increased sensitivity would be required before the routine use of SLND as an alternative to ALND could be recommended in this patient population.

\section{CONSIDERATIONS IN DATA INTERPRETATION}

Because Z1071 did not meet the prespecified end point, it is fair to conclude that SLND after neoadjuvant chemotherapy is not yet ready for "prime time." However, the question remains as to whether there is a way forward to be more selective in the axillary management in patients presenting with clinically node-positive disease.

In Z0171, the recommended technique for SLND was use of blue dye and radioisotope. A metaanalysis of studies evaluating SLND in early-stage breast cancer showed a higher SLN identification rate and lower FNR when dual tracers were used versus single-agent mapping. ${ }^{15}$ In the Z1071 trial, $79.1 \%$ of patients had mapping performed with dual tracers, $16.8 \%$ with radioisotope alone, and $4.1 \%$ with blue dye alone. For patients with dual tracer mapping, the FNR was $10.8 \%$, versus $20.3 \%$ when a single agent was used. The trial also specified that at least 2 SLNs be removed to ensure that surgeons do a complete 
SLND before beginning the ALND portion of the procedure. When the FNR was evaluated by the number of SLNs excised, it was $31.5 \%$ with one SLN removed (data presented at San Antonio Breast Cancer Symposium [SABCS] 2012), $21 \%$ with two SLNs removed, and $9.1 \%$ with three or more SLNs removed. ${ }^{7}$ Three or more SLNs were examined in $57.1 \%$ of patients.

These data are consistent with two other trials evaluating SLND in clinically node-positive patients treated with neoadjuvant chemotherapy. The Sentinel NeoAdjuvant (SENTINA) trial was a four-arm, prospective, multicenter trial evaluating SLND and ALND in patients receiving neoadjuvant chemotherapy. Arm $\mathrm{C}$ included patients that were clinically node positive who converted to clinically node negative after chemotherapy. FNRs were $24.3 \%$ when a single SLN was identified, $18.5 \%$ when two SLNs were removed, and less than $10 \%$ when three or more SLNs were removed. ${ }^{16}$ Similar to Z1071, the FNR was lower (8.6 \%) for patients who underwent mapping with radioisotope and blue dye. The Sentinel Node biopsy Following NeoAdjuvant Chemotherapy in biopsy-proven node-positive breast cancer (SN FNAC study) was presented at the 2013 American Society of Clinical Oncology meeting. ${ }^{17}$ The trial included 145 evaluable clinical T0-3, N1-2 patients undergoing SLND and ALND after neoadjuvant chemotherapy, and the FNR was $8.4 \%$. In that trial, SLNs that were negative by hematoxylin and eosin were evaluated by immunohistochemistry, and those with isolated tumor cells ( $\mathrm{pNOi+)}$ were considered positive. If the $\mathrm{pNO}+$ + were classified as negative, the FNR was $13.3 \%$. Similar to the Z1071 and SENTINA trials, identifying more SLNs resulted in lower FNRs; the FNR was $18.2 \%$ when one SLN was removed and $4.9 \%$ when two or more were excised. Taken together, the data from these trials regarding tracer use and the number of SLNs removed suggest that surgical technique is critical in lowering the FNR in this setting. Surgeons considering the use of SLND in node-positive patients receiving neoadjuvant chemotherapy should discuss the possibility that an ALND may be required if there is failure to identify an SLN or when only one SLN is identified.

\section{TARGETED AXILLARY DISSECTION}

After presentation of the Z1071 trial results, our breast surgical oncology group convened to discuss the data to determine how it should affect our practice. Although we recognize that the $12.6 \%$ FNR is high and that the clinical effect of a false-negative event in this population is unknown, we remain optimistic that there is a way forward to personalize axillary management in these node-positive patients, particularly those who have a good clinical response to neoadjuvant chemotherapy.
Data from Z1071 presented at the SABCS but not yet published suggested utility in placing a clip in the biopsyproven positive lymph node at diagnosis and then ensuring that the clipped node is removed as an SLN during the SLND procedure. In 96 patients in whom a clip was placed and documented to be one of the SLNs removed, the FNR was $7.4 \%$ (95\% confidence interval, 2.0-17.4\%). Taking this into consideration, we have proposed an approach to surgical management of the axilla in these clinically nodepositive patients receiving neoadjuvant chemotherapy termed targeted axillary dissection (TAD).

Briefly, TAD involves a multidisciplinary approach to axillary staging after neoadjuvant chemotherapy based on the finding that confirmation that one of the SLNs removed during SLND was the clipped node (i.e., the node with biopsy-proven metastasis) improved the FNR in Z1071. At the time of diagnosis, patients undergo an ultrasound examination of the breast and regional lymph nodes, and suspicious lymph nodes are subjected to fine-needle aspiration biopsy. ${ }^{18}$ For patients in whom only axillary nodes are abnormal in appearance, the most suspicious node is biopsied, and a clip is placed to mark that node so that it can later be targeted for removal. For patients with matted adenopathy (clinical N2) or disease identified in the internal mammary (clinical $\mathrm{N} 2$ if in the absence of clinical axillary disease or clinical N3 with clinically evident axillary disease), infraclavicular, or supraclavicular lymph nodes (clinical N3), no clip is placed, because these patients are either not eligible (clinical N3) or not included (clinical N2) in the primary analysis of Z1071, and they require ALND after completion of neoadjuvant chemotherapy. After completion of neoadjuvant chemotherapy, the patient undergoes SLND. In addition, we have the opportunity to localize the node with confirmed metastasis which contains a clip by using a wire or a $\mathrm{I}^{125}$ radioactive seed (Fig. 1), thereby enabling us to ensure removal of the clipped node. We have completed a pilot study showing that this technique is feasible and are currently evaluating efficacy in axillary staging after chemotherapy under an institutional review board-approved protocol. In addition to removing all SLNs and the clipped node, we are currently performing completion ALND. This will allow us to determine the rate of correlation between the SLN(s) and the clipped node and the FNR of TAD.

\section{MULTIDISCIPLINARY CONSIDERATIONS}

We have also discussed the Z1071 data in a multidisciplinary forum involving clinicians from surgical oncology, medical oncology, radiation oncology, radiology, and pathology. As a group, we agreed that optimizing axillary management for clinically node-positive patients is 
FIG. 1 Localization of a clipped lymph node with an $\mathrm{I}^{125}$ radioactive seed. At the time of breast cancer diagnosis, all patients undergo an ultrasound of the breast and regional nodal basins. Suspicious-appearing lymph nodes are subjected to fineneedle aspiration biopsy, and immediate assessment for specimen adequacy is performed by cytopathologists. A clip is placed in the biopsied lymph node. At the time of surgery, the clipped lymph node can be localized with a radioactive seed (a). When the seed-containing lymph node is removed during surgery, it is radiographed in the pathology suite to document the presence of the marker clip and radioactive seed (b)
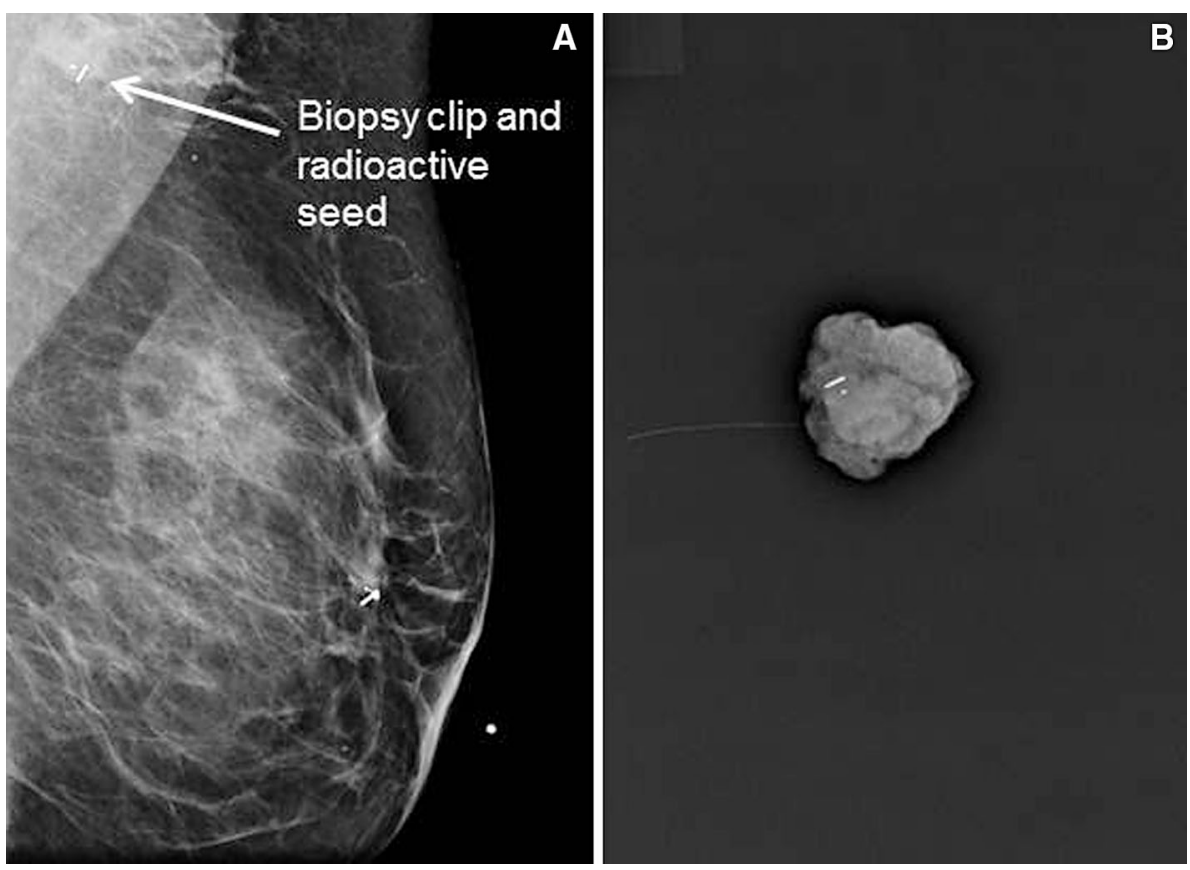

an important goal. For medical oncologists, the implication of Z1071 data is that in $12.6 \%$ of patients whom we declare to have a nodal pathologic complete response (pCR), there will be residual nodal disease. This may have prognostic implications but will not alter current therapeutic recommendations; patients with HER2-positive disease will complete 1 year of trastuzumab, and patients with hormone receptor (HR)-positive disease will receive endocrine therapy.

The implications of the FNR on adjuvant radiation therapy are more significant. For patients undergoing upfront surgery, recommendations for postmastectomy radiation therapy or regional nodal irradiation after breastconserving surgery are well defined. According to the National Comprehensive Cancer Network guidelines, nodal irradiation should be given for patients with 4 or more positive nodes and strongly considered for patients with 1-3 positive nodes. ${ }^{19}$ These recommendations are based on multiple randomized trials evaluating patients treated with upfront surgery. For patients receiving neoadjuvant chemotherapy, there are no randomized trial data to guide decisions regarding nodal irradiation; therefore, the indications are less clear. ${ }^{20,21}$ At MD Anderson, we routinely administer nodal irradiation after neoadjuvant chemotherapy to patients with clinical stage III disease and to most patients with residual positive nodes after chemotherapy. Regional nodal radiation is considered for select patients with clinical stage II disease, including those with high-risk features such as young age, estrogen receptornegative disease, lymphovascular invasion, and poor response to chemotherapy. ${ }^{20}$ The possibility of a falsenegative event therefore has significant implications for

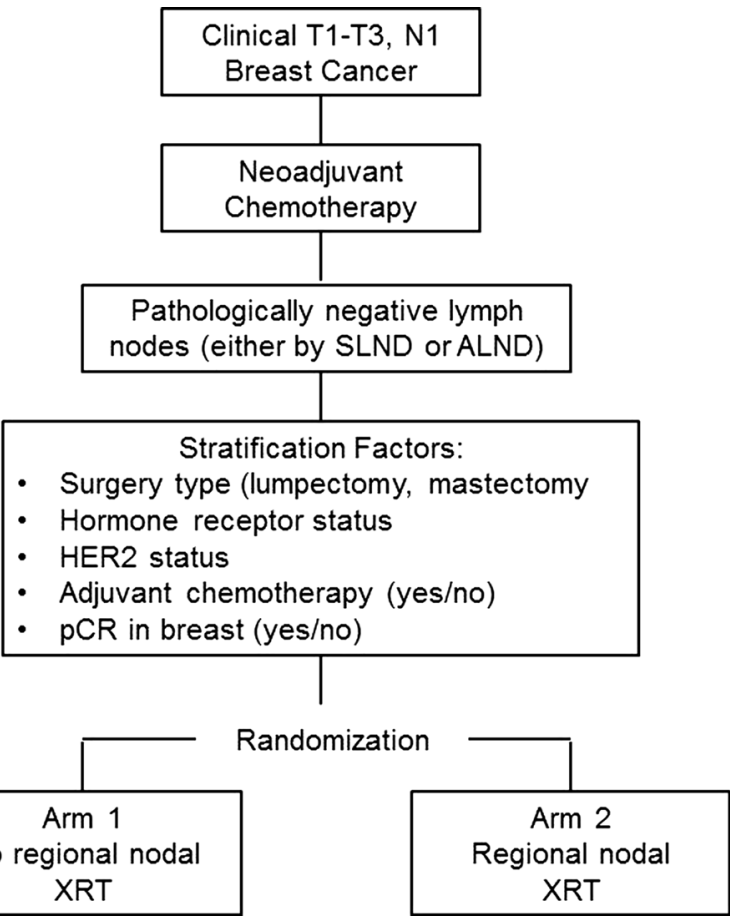

FIG. 2 Schema for the NSABP B-51/RTOG 1304 trial

patients with clinical stage II disease in whom our current practice at MD Anderson is to omit regional nodal radiation if they had no residual nodal disease identified in an ALND specimen. For patients with clinical stage II disease in whom only an SLND is performed and the SLN is found to be negative, our radiation oncologists would take into consideration the $12.6 \%$ FNR identified in Z1071 and the possibility of residual disease left behind when considering 

A11202 trial
FIG. 3 Schema for the Alliance

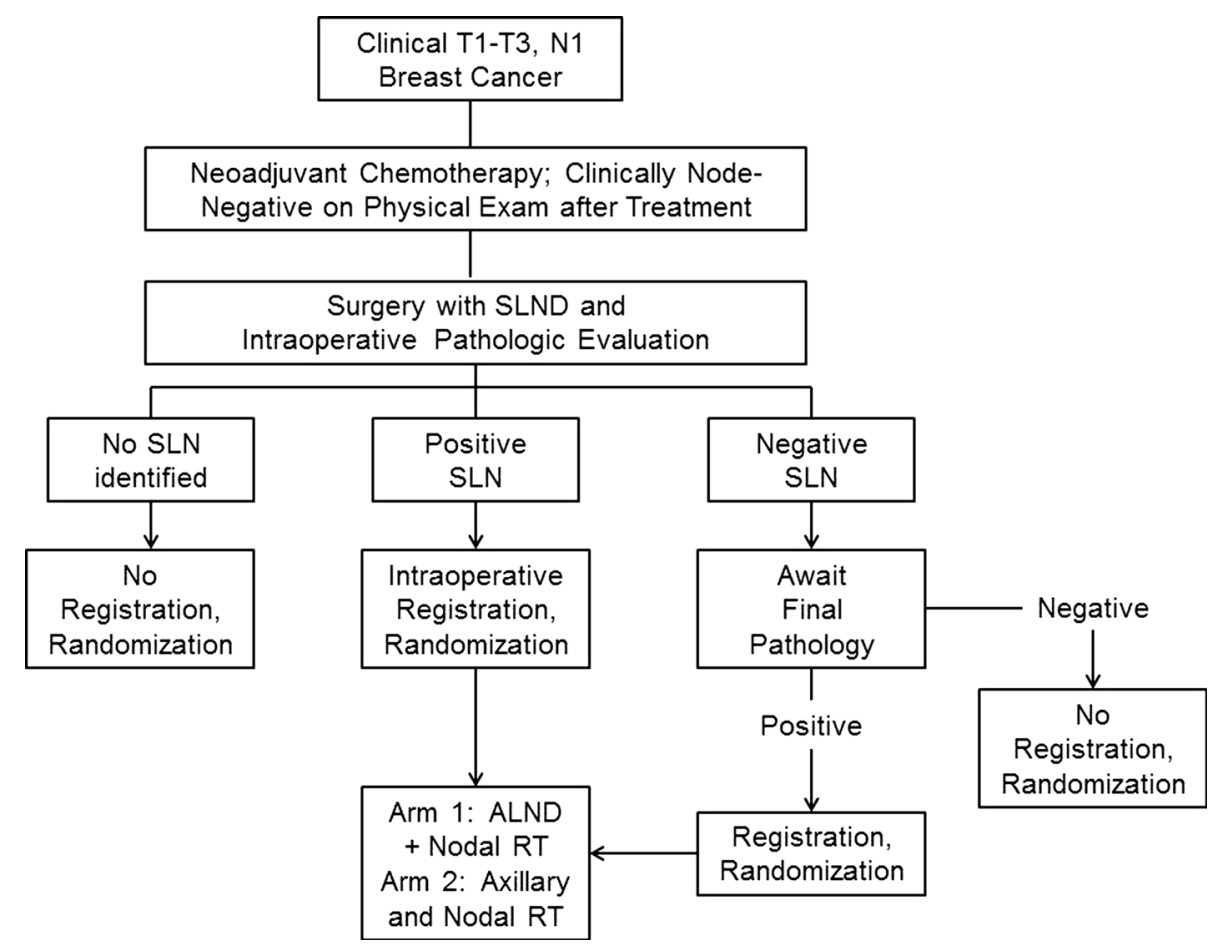

CONCLUSION

recommendations regarding regional nodal radiation. As will be discussed further below, optimal radiation for patients responding to neoadjuvant chemotherapy will be addressed in the ongoing NSABP/Radiation Therapy Oncology Group (RTOG) trial.

\section{CLINICAL TRIALS}

As the Z1071, SENTINA, and SN FNAC trials suggest, there is significant interest in determining optimal axillary management in patients presenting with clinically node-positive disease treated with neoadjuvant chemotherapy. This will be further addressed by two cooperative group trials, the NSABP B-51/RTOG1304 trial and the Alliance A11202 trial. The NSABP/RTOG trial randomizes clinically node-positive patients that convert to pathologically node-negative disease documented by SLND or ALND, to regional nodal irradiation or no regional nodal irradiation (Fig. 2). ${ }^{22}$ The primary aim is to evaluate whether the addition of regional nodal irradiation will significantly reduce the invasive breast cancer recurrence-free interval. The Alliance trial (Fig. 3) is randomizing patients with a positive SLN to ALND or axillary radiation. In addition to this axillary-specific treatment, all patients will receive nodal radiation targeting the supraclavicular and internal mammary lymph nodes in the first 3 intercostal spaces. ${ }^{23}$ The primary aim is to evaluate whether axillary radiation alone is not inferior to axillary radiation plus ALND in terms of invasive breast cancer recurrence-free survival.
The Z1071 trial failed to meet its prespecified threshold of an FNR $<10 \%$; however, important data from the study suggest a way forward for SLND in clinically node-positive patients receiving neoadjuvant chemotherapy. Surgical technique is critical, with the best results using dual tracers and ensuring all SLNs are removed. Experienced surgeons must assess the quality of the mapping procedure and determine whether SLND is appropriate in a given patient or whether ALND is warranted. TAD ensures that the node proven to be positive at diagnosis is clipped and removed at SLND and may help to minimize the FNR.

Selection of appropriate patients will also be important. Patients enrolled on Z1071 had an axillary ultrasound performed before and after chemotherapy, and those data are being analyzed to determine whether ultrasound can help select patients for SLND. Breast cancer subtype may also help guide patient selection. In the NeoSphere trial, there was a pCR rate of $45.8 \%$ in HER2-positive breast cancer patients treated with docetaxel, trastuzumab, and pertuzumab, with a $26.0 \%$ pCR rate in HR-positive patients and $63.2 \%$ in HR-negative patients. ${ }^{24}$ Similarly, in the ACOSOG Z1041 trial that investigated the effect of the timing of trastuzumab administration with anthracycline and taxane neoadjuvant chemotherapy in HER2positive patients, more than $70 \%$ of HR-negative, HER2positive patients experienced a pCR. ${ }^{25}$ Although these rates reflect pCR in the breast and the axilla, it is likely that nodal $\mathrm{pCR}$ rates are comparable. 
As continued improvements in systemic therapy lead to increased $\mathrm{pCR}$ rates, there will be further incentive to optimize local-regional management. SLND in patients with clinically node-positive disease receiving neoadjuvant chemotherapy should not be abandoned on the basis of the results of the Z1071 trial; rather, the data should guide future efforts to optimize local-regional management for these patients.

\section{REFERENCES}

1. Fleissig A, Fallowfield LJ, Langridge CI, et al. Post-operative arm morbidity and quality of life. Results of the ALMANAC randomised trial comparing sentinel node biopsy with standard axillary treatment in the management of patients with early breast cancer. Breast Cancer Res Treat. 2006;95:279-93.

2. Lucci A, McCall LM, Beitsch PD, et al. Surgical complications associated with sentinel lymph node dissection (SLND) plus axillary lymph node dissection compared with SLND alone in the American College of Surgeons Oncology Group Trial Z0011. J Clin Oncol. 2007:25:3657-63.

3. Julian TB, Anderson SJ, Krag DN, et al. 10-yr follow-up results of NSABP B-32, a randomized phase III clinical trial to compare sentinel node resection (SNR) to conventional axillary dissection (AD) in clinically node-negative breast cancer patients (abstract). J Clin Oncol. 2013;31(Suppl.):abstract 1000.

4. Xing Y, Foy M, Cox DD, Kuerer HM, Hunt KK, Cormier JN. Meta-analysis of sentinel lymph node biopsy after preoperative chemotherapy in patients with breast cancer. Br J Surg. 2006;93: 539-46.

5. van Deurzen CH, Vriens BE, Tjan-Heijnen VC, et al. Accuracy of sentinel node biopsy after neoadjuvant chemotherapy in breast cancer patients: a systematic review. Eur J Cancer. 2009;45:3124-30.

6. Hunt KK, Yi M, Mittendorf EA, et al. Sentinel lymph node surgery after neoadjuvant chemotherapy is accurate and reduces the need for axillary dissection in breast cancer patients. Ann Surg. 2009;250:558-66.

7. Boughey JC, Suman VJ, Mittendorf EA, et al. Sentinel lymph node surgery after neoadjuvant chemotherapy in patients with node-positive breast cancer: the ACOSOG Z1071 (Alliance) clinical trial. JAMA. 2013;310:1455-61.

8. Dominici LS, Negron Gonzalez VM, Buzdar AU, et al. Cytologically proven axillary lymph node metastases are eradicated in patients receiving preoperative chemotherapy with concurrent trastuzumab for HER2-positive breast cancer. Cancer. 2010;116: 2884-9.

9. Hennessy BT, Hortobagyi GN, Rouzier R, et al. Outcome after pathologic complete eradication of cytologically proven breast cancer axillary node metastases following primary chemotherapy. $J$ Clin Oncol. 2005;23:9304-11.

10. Shen J, Gilcrease MZ, Babiera GV, et al. Feasibility and accuracy of sentinel lymph node biopsy after preoperative chemotherapy in breast cancer patients with documented axillary metastases. Cancer. 2007;109:1255-63.

11. Newman EA, Sabel MS, Nees AV, et al. Sentinel lymph node biopsy performed after neoadjuvant chemotherapy is accurate in patients with documented node-positive breast cancer at presentation. Ann Surg Oncol. 2007;14:2946-52.

12. Classe JM, Bordes V, Campion L, et al. Sentinel lymph node biopsy after neoadjuvant chemotherapy for advanced breast cancer: results of Ganglion Sentinelle et Chimiotherapie Neoadjuvante, a French prospective multicentric study. J Clin Oncol. 2009;27:726-32.

13. Alvarado R, Yi M, Le-Petross $\mathrm{H}$, et al. The role for sentinel lymph node dissection after neoadjuvant chemotherapy in patients who present with node-positive breast cancer. Ann Surg Oncol. 2012;19:3177-84.

14. Mamounas EP, Brown A, Anderson S, et al. Sentinel node biopsy after neoadjuvant chemotherapy in breast cancer: results from National Surgical Adjuvant Breast and Bowel Project Protocol B27. J Clin Oncol. 2005;23:2694-702.

15. Kim T, Giuliano AE, Lyman GH. Lymphatic mapping and sentinel lymph node biopsy in early-stage breast carcinoma: a metaanalysis. Cancer. 2006;106:4-16.

16. Kuehn T, Bauerfeind I, Fehm T, et al. Sentinel-lymph-node biopsy in patients with breast cancer before and after neoadjuvant chemotherapy (SENTINA): a prospective, multicentre cohort study. Lancet Oncol. 2013;14:609-18.

17. Boileau J-F, Poirier B, Basik M, et al. Sentinel node biopsy following neoadjuvant chemotherapy in biopsy proven node positive breast cancer: the SN FNAC study (abstract). $J$ Clin Oncol. 2013;31(Suppl.):abstract 1018.

18. Krishnamurthy S, Sneige N, Bedi DG, et al. Role of ultrasoundguided fine-needle aspiration of indeterminate and suspicious axillary lymph nodes in the initial staging of breast carcinoma. Cancer. 2002;95:982-8.

19. National Comprehensive Cancer Network. 2014. Clinical practice guidelines in oncology: breast, version 1.2014. http://www.nccn. org/professionals/physician_gls/pdf/breast.pdf.

20. Hoffman KE, Mittendorf EA, Buchholz TA. Optimising radiation treatment decisions for patients who receive neoadjuvant chemotherapy and mastectomy. Lancet Oncol. 2012;13:e270-6.

21. Mamounas EP, Anderson SJ, Dignam JJ, et al. Predictors of locoregional recurrence after neoadjuvant chemotherapy: results from combined analysis of National Surgical Adjuvant Breast and Bowel Project B-18 and B-27. J Clin Oncol. 2012;30:3960-6.

22. Standard of comprehensive radiation therapy in treating patients with early-stage breast cancer previously treated with chemotherapy and surgery. 2014. http://clinicaltrials.gov/show/NCT 01872975.

23. Lymph node dissection and radiation therapy in treating patients with breast cancer previously treated with chemotherapy and surgery. $2014 \mathrm{http} / / /$ clinicaltrials.gov/show/NCT01901094.

24. Gianni L, Pienkowski T, Im YH, et al. Efficacy and safety of neoadjuvant pertuzumab and trastuzumab in women with locally advanced, inflammatory, or early HER2-positive breast cancer (NeoSphere): a randomised multicentre, open-label, phase 2 trial. Lancet Oncol. 2012;13:25-32.

25. Buzdar AU, Suman VJ, Meric-Bernstam F, et al. Fluorouracil, epirubicin, and cyclophosphamide (FEC-75) followed by paclitaxel plus trastuzumab versus paclitaxel plus trastuzumab followed by FEC-75 plus trastuzumab as neoadjuvant treatment for patients with HER2-positive breast cancer (Z1041): a randomised, controlled, phase 3 trial. Lancet Oncol. 2013;14:131725. 